\title{
CDISC ADaM Subject Trial Status Terminology
}

National Cancer Institute

\section{Source}

National Cancer Institute. CDISC ADaM Subject Trial Status Terminology. NCI Thesaurus.

Code C124296.

Terminology associated with the subject trial status codelist of the Clinical Data

Interchange Standards Consortium (CDISC) Analysis Data Model (ADaM). 